\title{
Childhood Renal Lymphangiectasia
}

To the Editor,

An asymptomatic 4 month-old white female had a $2 \times 3 \mathrm{~cm}$ faint birthmark on her mid-thoracic back consistent with a cutaneous telangiectasia. MRI of the spine incidentally demonstrated an infiltrative right renal lesion. Ultrasound showed an infiltrative lesion in the enlarged right kidney and proximal ipsilateral ureter as well as heterogeneously increased renal cortical echogenicity. Abdominal CT scan revealed a hypodense lesion (Hounsfield units from 0 to +20 ) infiltrating the renal pelvis and parenchyma, circumscribing the right kidney and proximal ureter (Figure-1, A). Delayed contrast images showed compression of the ureterovesical junction with medial displacement of the right ureter (Figure-1, B). Abdominal MRI characterized the lesion as heterogenously hyperintense and hypointense on $\mathrm{T} 2$ and $\mathrm{T} 1$ weighted images, respectively (Figure2 ). Right kidney differential function was $28 \%$ by MAG-3 renal scan. The infiltrative character of the lesion without significant enhancement is consistent with a renal lymphangiectasia. At 2.5 years of followup, she remains asymptomatic without radiological change.

Renal lymphangiectasia is a rare, benign condition characterized by developmental malformation of the perirenal lymphatic system. The lymphatic structures that surround the kidney fail to establish normal communication with the rest of the lymphatic system. The physiopathological process is ectasia of lymphatic vessels without obstruction $(1,2)$. It can be focal, unilateral or bilateral and may be found in pediatric or adult patients. There are reports of familial predisposition (2). Like other lymphatic lesions, renal lymphangiectasia can appear suddenly, grow rapidly, cease growth abruptly, or even regress spontaneously. Signs and symptoms may vary from none to microscopic or macroscopic hematuria, proteinuria, flank pain, abdominal pain/distension, palpable abdominal
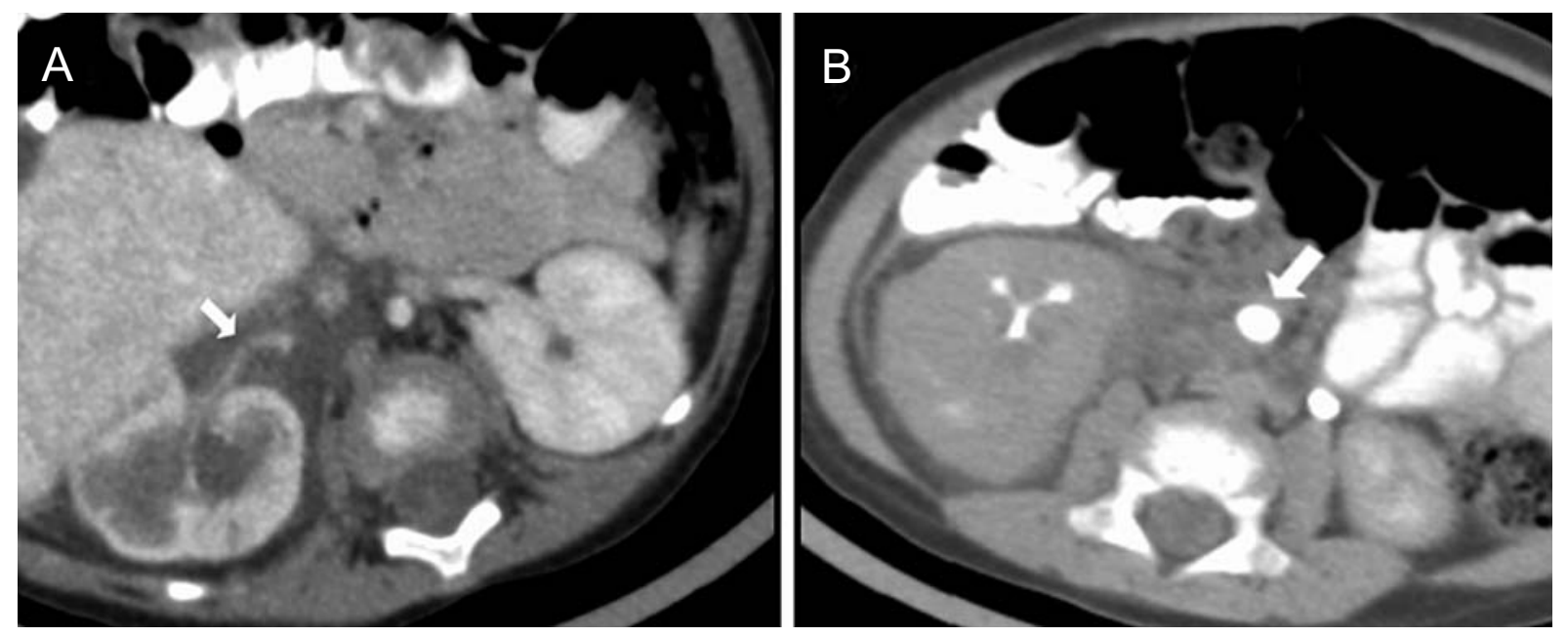

Figure 1 - A) An infiltrative, hypodense lesion is identified in the renal sinus, parenchyma and perirenal space. The right renal artery is circumscribed by the lesion (arrow). B) Delayed CT image shows elongated and distorted collecting system by the renal sinus mass. The right ureter is enlarged and displaced medially (arrow). 


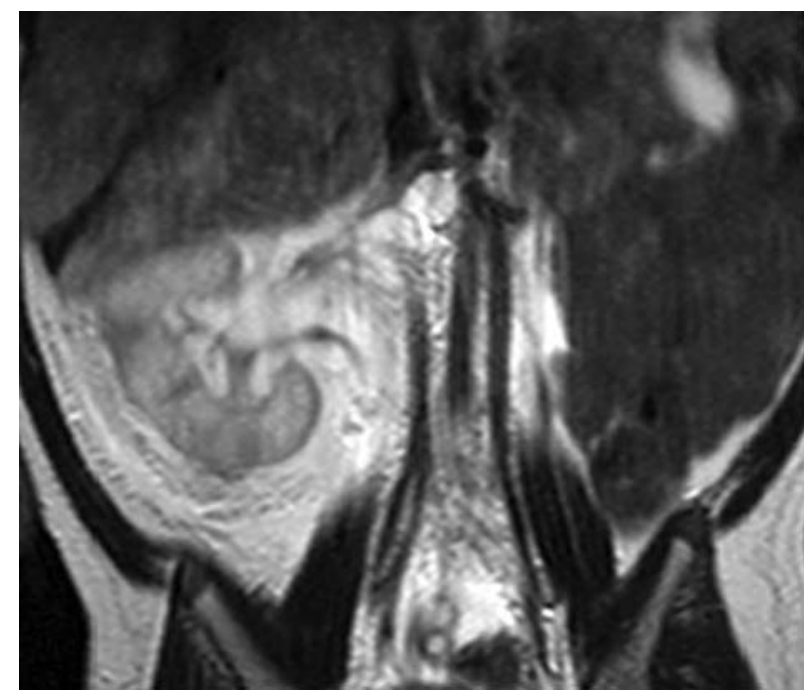

Figure 2 - Coronal T2 weighted image shows the mass in the renal sinus and perirenal space. The fluid content of the lesion is inferred from $T 2$ prolongation.

mass, lower extremity edema and hypertension. Renal function is generally preserved.

Radiological imaging modalities have aided diagnosis, including renal US, IVP, CT, and MRI. Renal US can show an enlarged and lobulated kidney with increased echogenicity and loss of normal corticomedullary differentiation (2). The CT scan reveals multilocular cyst/fluid filled masses with thin walls in the perirenal and parapelvic region. The MRI can show hyperintensity of the renal parenchyma especially at the cortical region and hypointensity at the medullary region. Also, multiple hyperintense lesions in perirenal spaces on T2-weighted images can be appreciated. Pediatric differential diagnosis includes polycystic renal disease, urinoma, renal lymphoma with perirenal involvement, renal tumors, etc. The diagnosis can be confirmed by needle aspiration of chylous fluid or by renal biopsy but multi-modal imaging is characteristic.

Management is often conservative due to the benign behavior of the lesion. Percutaneous drainage with administration of intravenous albumin and enteral medium chain triglycerides may be indicated if symptoms or ascites develop. Several cases of cyst decortication have resulted in nephrectomy due to uncontrolled intraoperative bleeding. While some suggest that asymptomatic patients with unchanged cystic lesions on US do not require follow-up, we feel patients should undergo lifelong follow-up as occasionally renal function will deteriorate.

\section{REFERENCES}

1. Varela JR, Bargiela A, Requejo I, Fernandez R, Darriba M, Pombo F: Bilateral renal lymphangiomatosis: US and CT findings. Eur Radiol. 1998; 8: 230-1.

2. Llorente JG, García AD, Sacristan JS, Chicharro GN: Renal lymphangiectasia: radiologic diagnosis and evolution. Abdom Imaging. 2002; 27: 637-9.

Fabian Sanchez, Juan C. Prieto, Korgun Koral \& Linda A. Baker Depart of Urology (FS, JCP, LAB) and Radiology (KK)

Children's Medical Center of Dallas University of Texas Southwestern Medical Center Dallas, Texas, USA E-mail: Linda.baker@utsouthwestern.edu 